\title{
Modelling of phase evolution during aluminizing processes
}

\section{Elisabetta Gariboldi, a , Marco Verani ${ }^{2, b}$, Christian Riva ${ }^{1, c}$}

\author{
${ }^{1}$ Politecnico di Milano, Dipartimento di Meccanica, Via La Masa 1, 20156 Milano (Italy) \\ ${ }^{21}$ Politecnico di Milano, Dipartimento di Matematica, P.zza L. da Vinci 32, 20133 Milano (Italy) \\ a elisabetta.gariboldi@polimi.it, b ${ }^{b}$ marco.verani@polimi.it, ${ }^{c}$ christian1.riva@mail.polimi.it
}

\begin{abstract}
Key words: Aluminizing, finite differences, free interface, phase evolution.
\end{abstract}
\begin{abstract}
Aluminizing processes are a well-known set of techniques industrially adopted to enrich in aluminum the surface layers of Ni-based alloys, thus improving their resistance to environmental interactions at high temperature. The results of aluminizing are described in terms of the presence, compositions and thickness of the sequence of the resulting surface diffusion layers. A combination of difficulties arising both from the mathematical and the material side restricted the number of available user-friendly models and their applicability to specific alloys or process conditions. The aim of the research work here presented is to overcome part of these difficulties. A synthesis of some well-established models was implemented in a robust numerical algorithm, that automatically prevents instabilities and convergence problems. Such numerical algorithm has been experimentally validated by comparing the results to the experimentally measured composition of profiles obtained for a set of vapor-phase aluminized samples of commercially pure Ni. The model was then applied to predict the effects of the process temperature and of the chemical composition of the surface.
\end{abstract}

\section{Introduction}

Aluminizing processes include a range of industrial techniques aimed at enriching in aluminum the surface of Ni-based alloys, thus improving their resistance to environmental high temperature interactions [1-4]. Their modelling can help carrying out industrial aluminizing processes by predicting the coating features under specific process routes. Further, the interaction of models with process control systems, can allow optimization or real-time adjustment of the process route on specific targets for the Al-enriched layered system.

Essential steps for developing a good physically-based model of a process are a correct description of the involved phenomena (leading not only to valid equations, but also to a clear identification of the key material and process parameters) and the availability of careful description of process results, useful for either validating the model or calibrating some parameters to specific cases.

The description of diffusion-controlled processes, such as aluminizing, is typically quantified in terms of the composition and thickness of the sequence of the resulting surface diffusion layers [46], sometimes completed by the weight gain and/or by the final position of phase interfaces. The solid phases to be taken into account for Al-enrichment process of $\mathrm{Ni}$ or Ni-base alloys at the relatively high process temperatures can be found in the Al-Ni phase diagram (such as that given in Fig.1a): solid solution of $\mathrm{Al}$ in $\mathrm{Ni}(\mathrm{Ni}), \mathrm{Ni}_{3} \mathrm{Al}, \mathrm{NiAl}$ and $\mathrm{Al}_{3} \mathrm{Ni}_{2}$. Depending on the temperature and/or on process conditions, one or more of these phases can be observed.

Concerning the physical description of phenomena involved in aluminizing, sets of chemical reactions and of gas- and solid-state diffusion processes are considered [7]. Further, since the rate of Al transport in processes involving gaseous transportation is high, aluminizing processes are typically controlled and can thus be described in terms of the solid-state diffusion of the Al deposited on the component surface [3,7]. In such processes, under equilibrium conditions, the activity of $\mathrm{Al}$ on the coating surface equals that of the Al-source material [3-4, 8-10]. It was also demonstrated that, at a given temperature, the surface composition of the component is related to that of the Al-source and that it can be considered as constant with time $[1,7,10]$.

For the above reasons, the key process parameters to be taken into account for process control or modeling are the time and the temperature at which the aluminizing occurs (the latter typically kept constant during the process). Further, Al activity, its deposition rate, composition of the source 
material, surface composition of the coating can be additionally taken into account. The key material parameters for the process are the substrate composition and the compositional ranges of the layered phases, as well as their interdiffusion coefficients. In the case of pure $\mathrm{Ni}$, that forms single-phase layers, the compositional ranges of the latter can be easily derived from equilibrium Al-Ni diagram. Diffusivity data for the same phases are also available in literature [1,11-14]. More complex structures, including non-homogeneous layers are possible in the case of Ni-alloy substrates and the identification of their compositional range is fairy complex $[15,16]$.

Several models have been developed and/or implemented during the years to describe the kinetics of intermetallic phase formation during aluminizing processes of nickel under the assumption that the controlling step of the process is the solid-state diffusion, as described earlier $[1,3,6,9,17]$. Many of them refer to a model presented in the mid 70's by Hickl and Heckel [1] describing the formation and movement of several phases in the uniaxial case. The model, hereafter referred as $\mathrm{HH}$ model, will be summarized in the next paragraph. The two authors proposed an approximated analytical formulation (parabolic growth of the layer thickness, well corresponding to experimental data after a transient period) at constant temperature and with constant surface and core compositions as well as for composition-independent diffusion coefficients. They also suggested a numerical method to develop solutions to the system of equations describing the diffusion process.

Some of the models of aluminizing proposed in the following years took into account the remarkable composition-dependence of the diffusion coefficient for the NiAl phase. This only change with respect to the $\mathrm{HH}$ model, introduced the need for numerical solutions, at least for equations referring to $\mathrm{NiAl}$ phase $[9,11]$. Nevertheless, the parabolic description of phase thicknesses changes, or of phase interface movements has often been assumed to model the results of aluminizing processes and/or to identify the material parameters $[4,6,17]$. These latter models can be easily applied only to define the coating characteristics obtained under stable process conditions (particularly, constant temperature, also reflected in a constant surface composition).

The aim of the research work here presented is to overcome such limitations by developing an implementation of the $\mathrm{HH}$ aluminizing model characterized by a robust numerical algorithm solving the whole set of partial differential equations. The method would offer the possibility to set even time- or (quasi-static) temperature-dependent boundary conditions in order to simulate more complex process operations. The first numerical algorithm validation tests and some examples of its application are presented in this paper.

\section{Mathematical model and numerical discretization}

Let us briefly describe the HH model [1], developed for the case of a semi-infinite medium. For simplicity reasons the names attributed by Hickl and Heckel to phases are here used (see Figure 1), even if some of the phases are currently referred in a different way and, more often, they are described in terms of their chemical composition. The aluminizing process has been characterized as semi-infinite, multi-phase, volume diffusion. Let $\mathrm{C}(\mathrm{x}, \mathrm{t})$ be the aluminum concentration at a given time $t$ at a given initial distance from the external surface $(x)$. Let $D_{i}$, with $i=1, \ldots, 4$ be the $i$-th phase interdiffusion coefficient and $\xi_{n}(t)$, for $n=0, \ldots, 4$ be the position at time $t$ of the $n$-th interphase interface. We assume $\xi_{4}(t)=\mathrm{L}>>1$ for every $\mathrm{t}$. Here $\mathrm{C}\left(\xi_{4}, \mathrm{t}\right)=\mathrm{C}_{0}$.

Let $\mathrm{J}_{\mathrm{i}}=-\mathrm{D}_{\mathrm{i}} \partial_{\mathrm{x}} \mathrm{C}$, for $\mathrm{i}=1, \ldots, 4$ be the flux in the $\mathrm{i}$-th phase. We set $\mathrm{J}_{0}=0$. The concentration $\mathrm{C}(\mathrm{x}, \mathrm{t})$ and the interphase-interface position $\xi_{i}(t)$, for $i=0, \ldots, 4$ are solutions to the following system of partial differential equations: for every time $t \in(0, \infty)$ there holds for $i=0, \ldots, 3$

$$
\begin{aligned}
& \frac{\partial C}{\partial t}=\frac{\partial}{\partial x}\left(D_{\mathrm{i}+1} \frac{\partial C}{\partial x}\right), \quad x \in\left(\xi_{\mathrm{i}}(t), \xi_{\mathrm{i}+1}(t)\right) \\
& \frac{\partial \xi_{\mathrm{i}}}{\partial \mathrm{t}}=\left(\frac{\mathrm{J}_{\mathrm{i}}-\mathrm{J}_{\mathrm{i}+1}}{\left(\mathrm{C}_{\mathrm{i}, \mathrm{i}+1}-\mathrm{C}_{\mathrm{i}+1, \mathrm{i}}\right)}\right)_{\mathrm{x}=\xi_{\mathrm{i}}(\mathrm{t})}
\end{aligned}
$$


a)

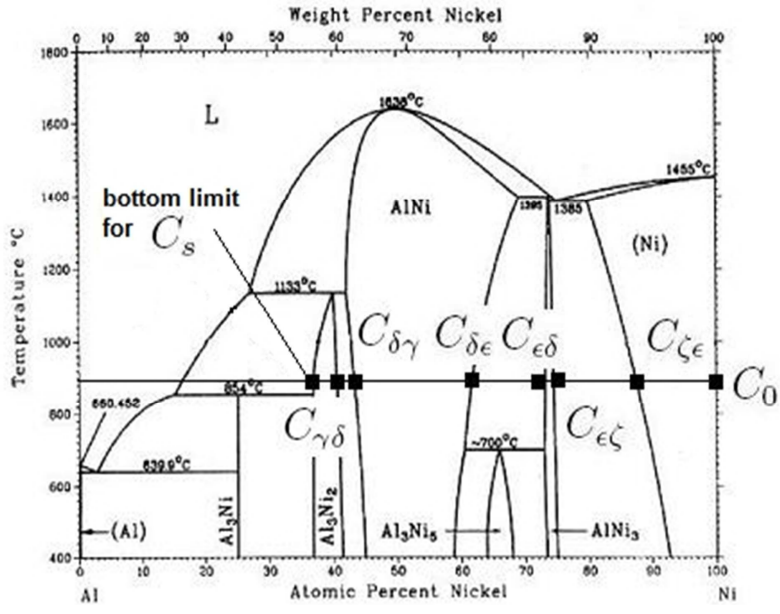

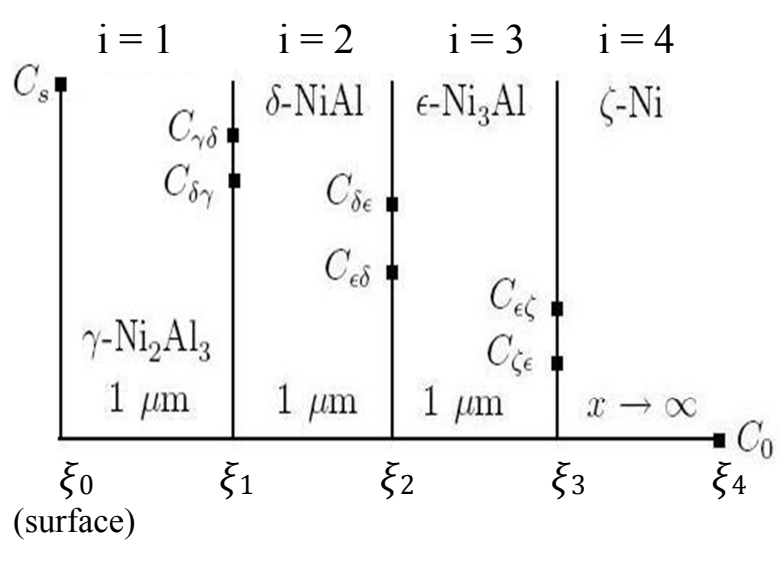

Figure 1: (a) Aluminum concentration $\mathrm{C}_{\mathrm{i}, \mathrm{j}}$ (expressed in at.\%) and upper modelled surface composition $\left(\mathrm{C}_{\mathrm{s}}\right)$ in the Ni-Al phase diagram. (b) identification of phases, interfaces and of their compositions in the 4-phase solid state diffusion model (b).

The above set of equations was supplemented by the following initial conditions at $t=0$ :

$$
C(x, 0)=C_{i+1, i+2}, \quad x \in\left(\xi_{i}(0), \xi_{i+1}(0)\right)
$$

These initial conditions correspond to the assumption that, at the beginning of the process, 4 layers exist. Their initial (small) thickness and homogeneous composition (the lower Al content allowed in equilibrium for the phase) are given.

The following Dirichlet boundary conditions were taken into account:

$$
\mathrm{C}\left(\xi_{i}(\mathrm{t})^{+}, \mathrm{t}\right)=\mathrm{C}_{\mathrm{i}+1, \mathrm{i}}, \mathrm{C}\left(\xi_{\mathrm{i}+1}(\mathrm{t})^{-}, \mathrm{t}\right)=\mathrm{C}_{\mathrm{i}+1, \mathrm{i}+2}
$$

where the values $\xi \mathrm{i}(0), \mathrm{i}=0, . ., 3$ are given. $\mathrm{C}_{\mathrm{i}, \mathrm{j}}$ are the chemical compositions of the phases at each side of an interface, corresponding to compositions of the solvus lines limiting the existence range phases in the Ni.Al phase diagram and to the surface composition $\left(C s=C\left(\xi_{0}(\mathrm{t})^{+}, \mathrm{t}\right)\right.$ see Figure 1). In the present implementation of the model such compositions and their related temperature dependence were derived by fitting data from [12,13] and are automatically computed by a numerical sub-routine once the temperature of the coating (assumed homogeneous through it) is known. On the other hand, $\mathrm{C}_{\mathrm{s}}$ represents the surface composition, for which, in general, slow evolution with time, can be taken into account.

The authors of the HH model applied it by using fixed values of interdiffusion coefficients $\mathrm{D}_{\mathrm{i}}$ to each phase, assigned for specific process temperatures. As already mentioned, the assumption of composition-independent $\mathrm{D}_{\mathrm{i}}$ was proved to be unsuitable for the case of NiAl phase. As a matter of fact, $D_{i}$ varies by more than 2 orders of magnitude within the composition range of this phase at temperatures of interest [12]. In addition to this, a relatively wide spread of experimental data exists for these coefficients, as recently discussed by [6]. Among the available data, those presented by [12] in the whole phase composition range at several temperatures ranging from 950 to $1150^{\circ} \mathrm{C}$ were fitted by obtaining a $D_{2}=D_{2}(T, C)$ correlation. As far as the other phases are concerned, their $D_{i}$ coefficients were assumed as composition-independent and temperature-dependent by means of Arrhenius-type laws, alternatively taken from or fitted on the basis of available literature data $[15,12-13]$.

Equations (1) and (2) were discretized by resorting to a classical explicit (in time) finite difference scheme, defining a suitable criterion for the accurate choice of the time step parameter to ensure the stability of the numerical system. The crucial point of the discretization was the handling of the computational domain, which varies in time, according to the velocities of the interphase-interfaces. 
Following [1], a finite difference discretization of the material derivative of the concentration $\mathrm{C}$

$$
\mathrm{D}_{\mathrm{t}} \mathrm{C}=\partial_{\mathrm{t}} \mathrm{C}+\mathrm{v} \partial_{\mathrm{x}} \mathrm{C},
$$

with $\mathrm{v}$ interface velocity, was applied. This allowed to adaptively map the concentration values at the spatial grid points of the old computational domain to the spatial grid points of the new computational domain. As a consequence, the spatial grid points were not equally spaced, but their positions were continuously adjusted as the interfaces moved (see Fig. 2). This resulted, in order to enforce the stability of the (explicit) numerical scheme, in a variable upper bound for time step. The correlation between temporal and spatial grids can lead under particular situations (such as the presence at a certain time of very thin layers) to an excessive reduction of time step, causing long lasting simulations of the process. Thus, a minimum time and spatial step can be specified for each model run. An unconditionally stable implicit method of discretization was provided as an alternative choice.
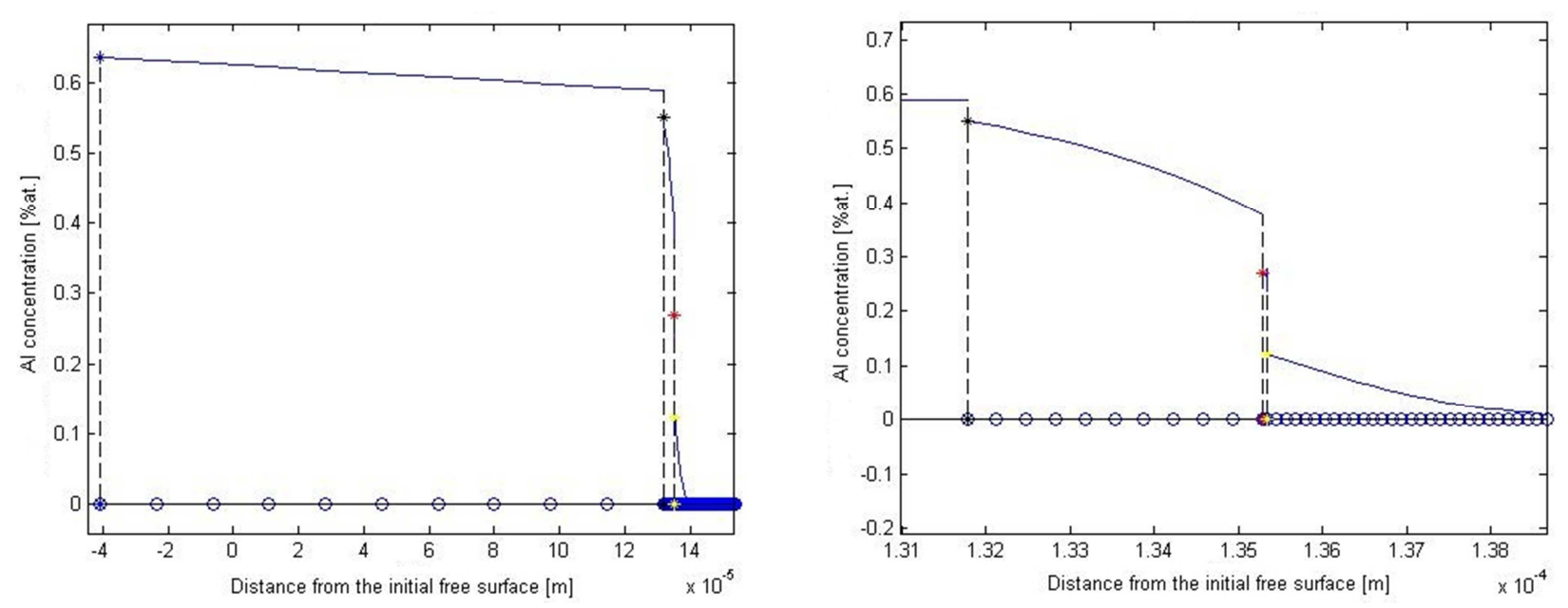

Figure 2. Example of the chemical profile supplied as model result, here shown with different $\mathrm{x}$ scales. It reproduces the high-Al activity aluminizing process $\left(4\right.$ hours at $870^{\circ} \mathrm{C}$ ) modelled by [1]. The same plots also show that at a given time, the distance between points of the spatial mobile grid is different for each layer.

\section{Experimental validation of the model}

The model was tested using both material data and initial/boundary conditions of $[1,11]$ and comparing the obtained results to those presented in the papers. The stability of the model and of its space or time adjustment rules were tested by modeling the case of a first step in a two-phase lowtemperature and high-activity aluminizing process, being it relatively complex from the mathematical point of view (Fig. 2). The material properties in this test were those given in papers. Further, an experimental validation of the model was set up. Low carbon commercially pure nickel (UNS N02201, with $\mathrm{Ni}=99.0 \mathrm{wt} \%$ min and $\mathrm{C}=0.02 \mathrm{wt} \% \max$ ) was chosen as substrate material. A set of $30 \mathrm{X} 10 \mathrm{X} 4.5 \mathrm{~mm}^{3}$ samples were cut and their surfaces were metallographically polished. They were then vapor phase aluminized for different holding times under the same process conditions (at a temperature of $1050^{\circ} \mathrm{C}$ ). SEM analyses combined with EDS microanalyses were performed on the external surface as well as on metallographic section of the specimens in order to derive the main microstructural features and composition profiles of the aluminized specimens. Joined EBSD analyses confirmed the identification of phases.

The surface of aluminized samples revealed grain boundaries in relief with respect to the grain interior, as typically found in low-Al activity aluminized parts, where during the process external Al-Ni layer forms and outward diffusion of Ni prevails [10]. Microanalyses confirmed the relative stability of the surface composition. Below the external phase, the presence of a thin single-phase $\mathrm{Ni}_{3} \mathrm{Al}$ layer and of some voids at its interface with the (Al-enriched) Ni clearly appeared from 
metallographic sections (Fig. 3a). The Al-content profiles (Fig. 3b) clearly show the interface between phases and compositional gradients within them. Under the same temperature and for a given surface composition, the model described the progressive movement outward of the external surface, while internal interfaces moved towards the core of the component. In the initial stage of the process, the model suggested a faster inward movement of the $\mathrm{NiAl} / \mathrm{Ni}_{3} \mathrm{Al}$ interface with respect to the $\mathrm{Ni}_{3} \mathrm{Al} / \mathrm{Ni}$ interface, and the initially modelled $1 \mu \mathrm{m}$ thick $\mathrm{Ni}_{3} \mathrm{Al}$ layer thinned. After this transient period, lasting less than a minute, the thickness of this phase began to grow. After 5 hours the thickness of $\mathrm{Ni}_{3} \mathrm{Al}$ was about $3 \mu \mathrm{m}, 1 / 10$ that of $\mathrm{NiAl}$.

a)

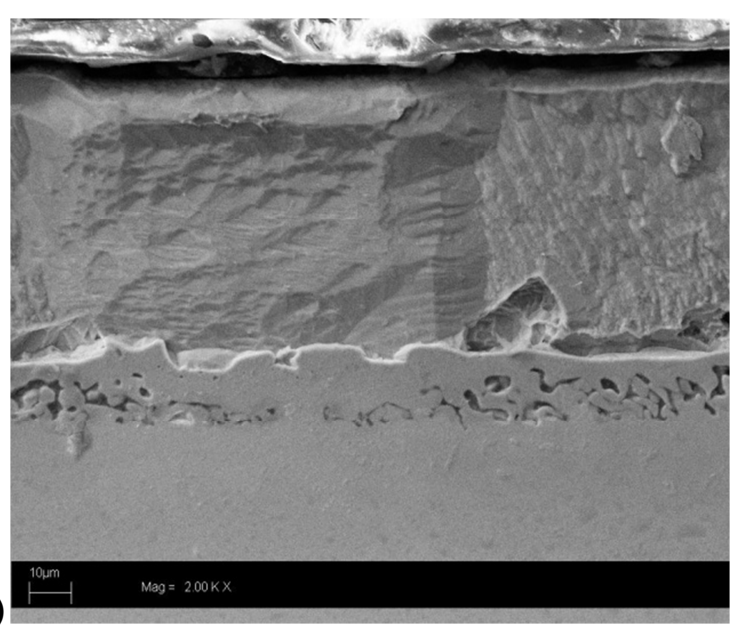

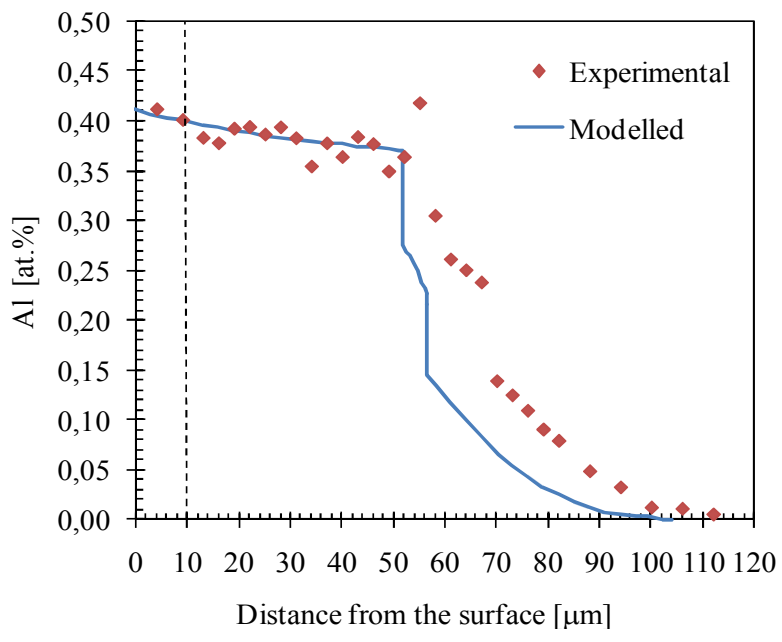

Figure 3. a) SEM macrograph of a metallographic section of an aluminized specimen. b) Modelled and experimental composition profiles at corresponding aluminizing times. The dashed vertical line represents the initial position of the external surface.

The modelled profiles were compared with the experimental ones at corresponding process conditions, as shown in Fig. 3b. The compositions at interfaces are in agreement with those taken into account in the model. The measured $\mathrm{Ni}_{3} \mathrm{Al}$ thickness roughly corresponded to the modelled one, while that of the external phase was slightly underestimated by the model.
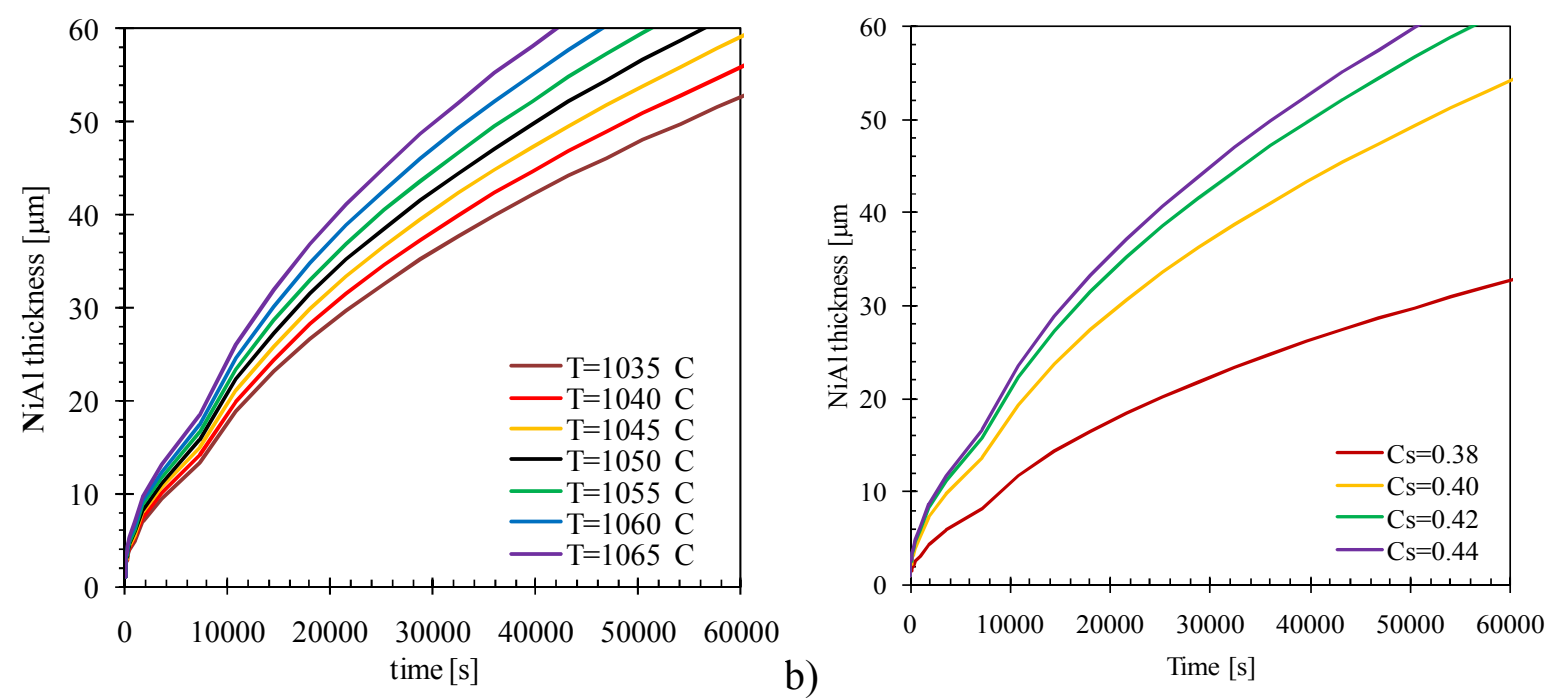

Figure 4. Modelled evolution of NiAl layer thickness under various temperatures (a) and surface Al contents (b). 


\section{An application of the model: evaluation of sensitivity of aluminizing process}

A first application of the model was the evaluation of the sensitivity of the coating features to process conditions. Among these, the attention was focused on the time, temperature and surface $\mathrm{cm}$ position. The effect of temperature was estimated by repeatedly running the model with a fixed surface composition of $42 \mathrm{Al}$ at $\%$ and temperatures in the range $1035-1065^{\circ} \mathrm{C}$. The results are here presented in Fig. $4 \mathrm{a}$ in terms of the thickness of $\mathrm{NiAl}$ phase. It can be easily seen that a temperature variation of $5^{\circ} \mathrm{C}$ (typical tolerance of temperature sensors in industrial plants operating at about $1000-1100^{\circ} \mathrm{C}$ ), led to a difference of thickness of the NiAl phase formed after $5 \mathrm{~h}$ of about $1.5 \mu \mathrm{m}$ (4.5\%). The $\%$ difference caused by $5^{\circ} \mathrm{C}$ variation increased as process temperature rose or process time was longer. The effect of the surface composition was estimated similarly, varying $\mathrm{C}_{\mathrm{s}}$ at a fixed temperature of $1050^{\circ} \mathrm{C}$. As shown in Fig. $4 \mathrm{~b}$, the thickness of NiAl progressively tends to be less sensitive to $\mathrm{C}_{\mathrm{s}}$ as it increases towards the value for minimum interdiffusivity. Correspondingly, the $\mathrm{Al}$ concentration gradient at surface in NiAl phase progressively increased.

\section{Conclusions}

An established solid-state diffusion-based model of aluminizing processes was implemented in a robust numerical algorithm in view of describing the layered structure evolution during processes involving relatively slow temperature or surface compositional changes. The model automatically prevents instabilities and convergence problems. The numerical algorithm has been experimentally validated by comparing results to the composition profiles obtained for a set of vapor-phase aluminized samples of commercially pure Ni. The model was then applied to estimate the effect of the process temperature and of the surface $\mathrm{Al}$ content on the composition profiles of the coating. Several modification of the base aluminizing process can be described by simply introducing the time/temperature/composition dependence of material parameters and boundary conditions.

\section{References}

[1] A.J. Hickl, R.W. Heckel: Metall. Trans., Vol. 6A (1975), p.431.

[2] G.W. Goward: Surf. Coat. Technol, Vol. 108-109 (1998), p.73.

[3] N. Voudouris, Ch. Christoglou, G.N. Angelopoulos: Surf. Coat. Technol., Vol. 141 (2001), p. 275.

[4] J. Kohlscheen, H.R. Stock: Surf. Coat. Technol., vol. 202 (207), p. 613.

[5] J.T. John, R.S. Sinivasa, P.K. De: Thin Solid Films, Vol. 466 (2004), p. 339.

[6] H. Wei, X. Sun, Q. Zheng, H. Guan, Z. Hu: Acta Mater., Vol. 52 (2004), p. 2645

[7] J.T. John, G.B. Kale, S.R. Bhardwaj, R.S. Sinivasa, P.K. De: Thin solid films, Vol. 466 (2004), p. 331.

[8] R.Sivakumar, L.L.Seigle: Metall. Trans., Vol. 7A (1976), p. 1073.

[9] B.K. Gupta, A.K. Sarkhel, L. Seigle: Thin Solid Films, Vol. 39 (1976), p. 131.

[10] W-P Sun, H.J. Lin, M-H Hon: Metall. Trans, Vol. 17A (1986), p. 215.

[11]A. Sarkhel, L.L. Seigle: Metall. Trans., Vol. 7A (1976), p. 899.

[12]T. Helander, J. Agren: Acta Mater., Vol. 47 (1999), p. 1141.

[13] C Cserati, A. Paul, A.A. Kodentsov, M.J.H. van Dal, F.J.J. van Loo: Intermetallics, vol. 11 (2003), p. 291.

[14]K. Fujiama, Z. Holta: Acta Materialia, Vol. 50 (2002), p. 1571.

[15]H. Wie, H.Y. Zhang, G.C. Hou, X.F. Sun, M.S. Durgusch, X. Yao, Z.Q. Hu: J. alloys and compounds, Vol. 481 (2009), p. 326.

[16]D.M. Das, V. Singh, S.V. Josh: Metall. Mater. Trans., Vol. 29A (1998), p. 2173.

[17] V.M. Garcia. P.M. Mors, C. Schwerer: Acta Mater., Vol. 48 (2000), p. 1201. 